\title{
Resolution of Mass Effect and Compression Symptoms following Endoluminal Flow Diversion for the Treatment of Intracranial Aneurysms
}

\author{
I. Szikora, M. Marosfői, B. Salomváry, Z. Berentei, and I. Gubucz
}

\begin{abstract}
BACKGROUND AND PURPOSE: Alleviation of aneurysm induced mass effect has been difficult with both conventional endovascular and surgical techniques. Our aim was to study the efficacy of endovascular flow modification on aneurysm-induced mass effect and compression syndrome, as demonstrated by cross-sectional imaging studies and clinical follow-up.
\end{abstract}

MATERIALS AND METHODS: Thirty aneurysms larger than $10 \mathrm{~mm}$ were treated by flow diversion alone and previously had undergone pre- and posttreatment cross-sectional imaging. Pretreatment MR imaging or contrast CT, follow-up angiography at 6 months, and follow-up MR imaging studies between 6 and 18 months were retrospectively analyzed. The neurologic and neuro-ophthalmologic statuses of all patients were recorded before treatment and at the time of follow-up cross-sectional imaging.

RESULTS: At 6 months, 28 aneurysms were completely occluded, 1 had a neck remnant, and 1 had residual filling on angiography. Between 6 and 18 months, 3 aneurysms decreased in size and 27 completely collapsed as demonstrated on MR imaging. Before treatment, 6 patients had vision loss, 10 had double vision due to a third or sixth nerve palsy or both, and 1 had hemiparesis due to brain stem compression. On MR imaging follow-up, vision loss had either improved or resolved in all except 1 patient, double vision had resolved completely (7/10) or partially (3/10), and the patient with brain stem compression became asymptomatic. There was no bleeding observed in this series. One parent artery thrombosis resulted in a major infarct.

CONCLUSIONS: Endovascular flow diversion is a highly effective technique for resolving radiologic mass effect and clinical compression syndromes.

ABBREVIATIONS: FD = flow diverter; GP IIb/IIla = glycoprotein Ilb/Illa; PED = Pipeline Embolization Device; PUFS = Pipeline for Uncoilable or Failed Aneurysms

arge and giant intracranial aneurysms pose an increased health -risk due to a high incidence of rupture ${ }^{1,2}$ and resultant neurologic deficits caused by compression of neighboring neural structures. ${ }^{3}$ Successful treatment of aneurysms presenting with mass effect is limited by high surgical morbidity ${ }^{4,5}$ and low endovascular efficacy, due to a high rate of incomplete occlusion and recurrence. ${ }^{6,7}$ Parent artery occlusion is effective but restricted to aneurysms with sufficient collateral circulation. ${ }^{8,9}$ Surgical bypass can potentially compensate for the lack of collaterals but adds substantial morbidity. ${ }^{10,11}$ Modification of flow within the parent

Received December 28, 2012; accepted after revision February 5, 2013.

From the Departments of Neurointerventions (I.S., M.M., Z.B., I.G.) and Neuroophthalmology (B.S.), National Institute of Clinical Neurosciences, Budapest, Hungary.

This work was supported in part by a grant from OTKA, No. T 73773 and, in part, by a research grant from Covidien/ev3.

Please address correspondence to Istvan Szikora, MD, National Institute of Neurosciences, Amerikai ut 57, H-1145 Budapest, Hungary; e-mail: h13424szi@ella.hu

- Indicates open access to non-subscribers at www.ajnr.org

http://dx.doi.org/10.3174/ajnr.A3547 artery is a method that attempts to reduce intra-aneurysmal circulation, leading to aneurysm thrombosis. Reports published to date demonstrate a very high rate of complete and stable occlusion of aneurysms that were considered difficult or impossible to treat by conventional endovascular techniques. ${ }^{12-17}$ Collapse of large and giant aneurysms has also been observed. ${ }^{13}$ The purpose of this work was to analyze the effect of flow modification on the radiologic mass effect and clinical compression syndromes induced by large and giant aneurysms.

\section{MATERIALS AND METHODS}

Ninety-eight aneurysms in 81 patients were treated at a single center with flow diversion by using either the PED (Covidien/ev3, Irvine, California) (97 aneurysms) or the Silk stent (Balt Extrusion, Montmorency, France) (1 aneurysm) from 2006 to 2011. All subjects were treated under institutional review board-approved protocols as part of the Pipeline Embolization Device for the Intracranial Treatment of Aneurysms Trial study, ${ }^{15}$ the PUFS study, ${ }^{18}$ or the Reconstructive Aneurysm Treatment study (un- 


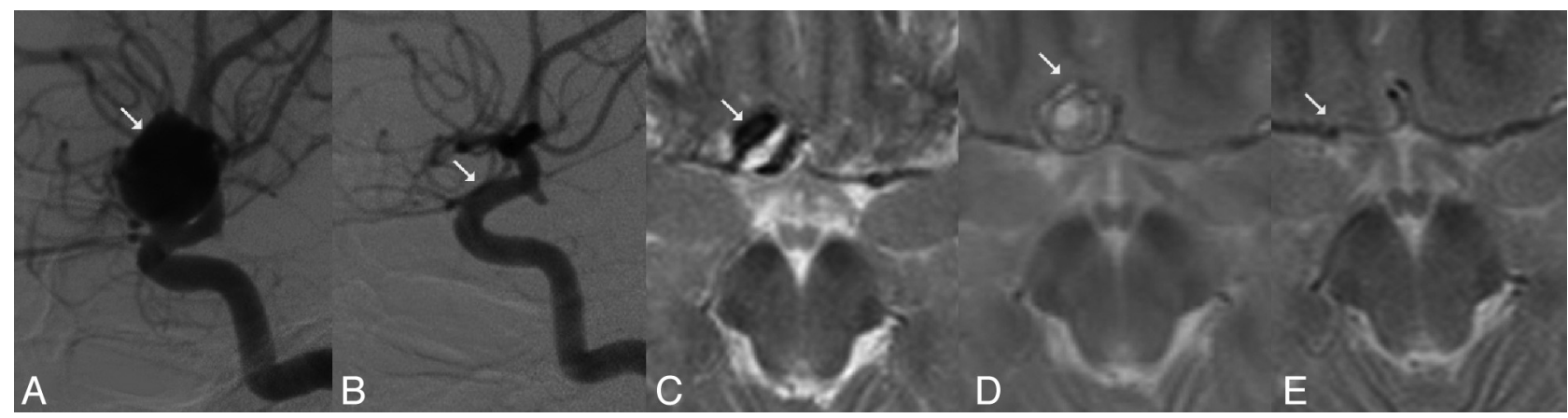

FIG 1. Complete angiographic occlusion of a paraophthalmic aneurysm and aneurysm collapse following FD treatment. A, Large paraophthalmic aneurysm (arrow) causing optic nerve neuropathy on DSA, lateral view. The patient's visual acuity was 0.4 . B, Complete angiographic occlusion of the aneurysm and reconstruction of the parent artery (arrow) 6 months after treatment with a PED. The patient's visual acuity was 0.5 . C, The same aneurysm demonstrated by T2WI with mass effect (arrow) and mixed signal intensity. D, Lack of signal void within the aneurysm on T2WI in the same patient 2 months later indicates aneurysm thrombosis. The size of the aneurysm and related mass effect remain unchanged (arrow). Optic nerve neuropathy progressed; the patient's visual acuity was 0.1 . E, T2WI in the same patient demonstrates aneurysm collapse and elimination of mass effect at 18 months after treatment. The patient's visual acuity at this point was 0.9 .

published); informed consent was obtained from all subjects. To properly evaluate the effect of flow diversion alone, we excluded from the current analysis aneurysms that had coils in their sacs because they were either previously treated or packed during the FD treatment. For the purpose of this study, the aneurysms that were selected had a diameter larger than $10 \mathrm{~mm}$, were treated by flow diversion alone (ie, without coils), and previously had the following imaging studies: cross-sectional imaging either by MR imaging or contrast CT before treatment; follow-up angiography at 6 months; and T1WI, T2WI, and time-of-flight MRA between 2 and 18 months. Twelve aneurysms were studied by MR imaging at 3 months, and all, between 12 and 18 months after treatment. All these patients had a neurologic and/or neuro-ophthalmologic evaluation before treatment and at the time of follow-up crosssectional imaging. Clinical and imaging records were evaluated retrospectively.

\section{RESULTS}

Thirty aneurysms were identified in 27 patients.

Indications. One patient was treated for an incidentally discovered aneurysm. Twelve patients presented with headaches, and 17 , with neurologic symptoms related to their aneurysms. Among those, 1 patient had hemiparesis due to brain stem compression, 6 had vision loss of different degrees related to optic nerve or chiasm compression, and 10 had diplopia related to either third $(n=$ $3)$ or sixth $(n=2)$ cranial nerve palsy or both $(n=5)$.

\section{Aneurysm Characteristics}

Twenty-nine aneurysms were located on the ICA, and 1, on the basilar trunk. ICA aneurysms either involved the cavernous $(n=$ $10)$ or the paraophthalmic $(n=16)$ segment of the ICA or arose at the origin of either the superior hypophyseal artery $(n=1)$ or the posterior communicating artery $(n=1)$. Sixteen aneurysms had a diameter between 10 and $20 \mathrm{~mm}, 12$ measured $20-30 \mathrm{~mm}$, and 2 were $\geq 30 \mathrm{~mm}$ in diameter. The mean aneurysm size was 18.9 $\mathrm{mm}$, and the mean neck size was $9.7 \mathrm{~mm}$. Three of the 30 aneurysms were fusiform. Two aneurysms of the ICA demonstrated calcifications in their wall on CT.

\section{Procedural Technique}

All patients were pretreated with oral aspirin (either 100 or 300 $\mathrm{mg}$ /day) and clopidogrel (75 mg/day) for a minimum of 4 days before the procedure. All subjects were intravenously heparinized during the endovascular procedure to reach an activated clotting time of twice their baseline level. No additional heparin was used following the procedure, except in cases of intraprocedural thrombotic complications.

For flow diversion, the PED (Covidien/ev3) was used in 29 aneurysms, and a combination of a LEO and a Silk stent (Balt Extrusion), in 1 case. A single-layer FD was used in 1 case (the aneurysm was located at the posterior communicating artery origin), and the aneurysm neck was covered with 2 layers in 18 cases. Three coaxial layers were used in 8 cases, and 4 layers, in 1 case. A long FD construct was built to cover the lengths of fusiform aneurysms by using 5 pieces in one case and 15 pieces in another case. All patients were maintained on double antiplatelet treatment for 1.5-3 months. Steroids were not routinely administered perioperatively, but subjects with aggravated postprocedure symptoms received methylprednisolone, $2 \times 250 \mathrm{mg} /$ day for $2-3$ days, with a gradually tapering dose for an additional 10-14 days.

\section{Complications}

As a technical complication, incomplete vessel wall apposition was observed in 1 case and was corrected with balloon dilation. In-stent thrombosis occurred during the procedure in 2 cases and was resolved by thrombolysis with application of both tissue plasminogen activator and GP IIb/IIIa inhibitor in one case and GP IIb/IIIa inhibitor only in another case. Subacute occlusion of the parent artery occurred in 1 case 2 days postprocedure, resulting in transient hemiparesis. This was not treated due to good collateral circulation, and it later recanalized spontaneously. Delayed occlusion resulting in hemiparesis was found in 1 case 3 months after treatment. Overall, transient neurologic deficit occurred in 2 patients, and a permanent deficit occurred in 1 patient.

\section{Angiographic Follow-Up}

All aneurysms were followed by angiography at 6 months. In addition, 13 aneurysms underwent follow-up angiographies at 1 


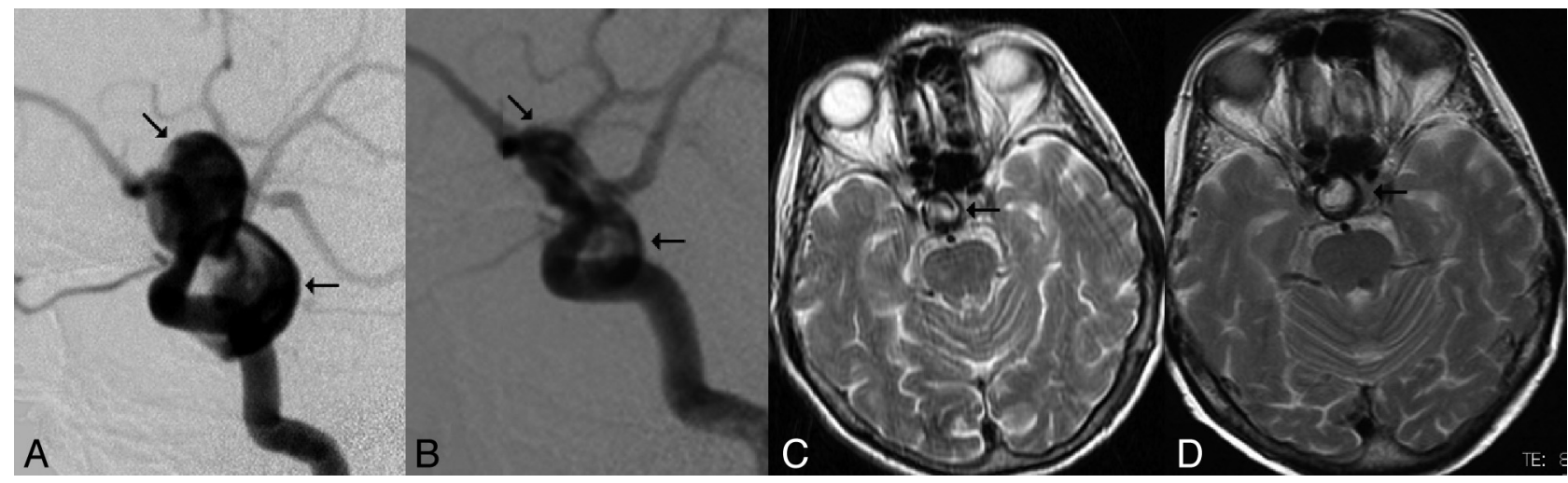

FIG 2. Incomplete angiographic occlusion and remaining mass effect following FD treatment. A, A large bilobulated paraophthalmic aneurysm (arrows) causing optic nerve compression as demonstrated by DSA, lateral view. B, DSA, lateral view 6 months after PED treatment. The arrow indicates residual filling in the lower lobule of the aneurysm. C, T2WI shows mixed signal intensity inside the aneurysm (arrow) before treatment. The patient's visual acuity was 0.2. D, T2WI 18 months after treatment demonstrates high T2 signal inside the aneurysm. The low-signal rim was related to aneurysm wall calcification, and the aneurysm size remained unchanged. The patient's visual acuity deteriorated slightly (0.1).

year, 12 due to the follow-up protocol of the PUFS study and 1 because the 6-month angiogram demonstrated a nonsignificant clinically silent narrowing of the parent artery. At 6 months, 28 (93.3\%) aneurysms were completely occluded (Fig 1A, -B), 1 $(3.3 \%)$ had partial aneurysm filling (Fig $2 A,-B)$, and $1(3.3 \%)$ had a neck remnant. Of the 13 aneurysms that had 1-year follow-up angiography, 12 (92.3\%) had complete angiographic occlusion and $1(7.7 \%)$ had a neck remnant.

\section{MR Imaging Follow-Up}

The last follow-up MR imaging study between 2 and 18 months demonstrated complete collapse of the aneurysm in 27 cases (Fig $1 C,-D)$ and reduction of the aneurysm size in 2 cases ( 9 and 18 months posttreatment), and the aneurysm remained unchanged in 1 case at 18 months (Fig $2 C,-D$ ). Regardless of the timing of the study, in aneurysms that did not yet fully collapse, no flow signal was detected by the time-of-flight MRA, but high signal intensity was found on the T2-weighted images within the aneurysm sac (Fig 2).

\section{Clinical Outcomes}

Four patients had temporary aggravation of their symptoms requiring steroid therapy. One had worsening headaches 2 weeks after treatment, which lasted for 3 weeks. Two patients had further deterioration of visual acuity 8 weeks later (Fig 1), and 1 additional patient exhibited progression of her third cranial nerve palsy and a new sixth cranial nerve palsy 5 days postprocedure. All these patients had their aneurysms on the cavernous or paraophthalmic section of the carotid artery. Despite aggressive steroid therapy, these aggravated cranial nerve symptoms only started improving after 6 months. Altogether, among the 6 patients presenting with vision loss, the mean visual acuity improved from 0.3 to 0.8 on a scale of $0.0-1.0$ by the last follow-up. In 3 cases, vision as low as $0.1-0.4$ normalized to 1.0. In another 2 cases, vision improved from as low as finger counting only to quantifiable vision of 0.6 and from 0.3 to 0.8 . In 1 case in which the aneurysm was incompletely occluded on angiography and did not collapse as seen on MR imaging, the patient's visual acuity slightly deteriorated from 0.2 to 0.1 (Fig 2). All 10 subjects who had presented with diplopia improved: Seven were completely cured, and 3 had minor diplopia at the time of the last MR imaging follow-up. Two of the minor diplopia cases were related to a residual partial third nerve palsy, and 1 was caused by residual dysfunction of both the third and sixth nerves. Hemiparesis in 1 patient presenting with brain stem compression was relieved completely by 6 months after treatment.

\section{DISCUSSION}

Large and giant aneurysms represent a challenge for both surgical and endovascular approaches. Such aneurysms often present with mass effect and corresponding compression syndrome on the neighboring neural tissue. Therefore, the goal of treatment is not only to prevent rupture but also to reduce the pulsating mass and eliminate the associated mass effect. Unfortunately, this goal is difficult to achieve by conventional endovascular techniques. Packing of the aneurysm with soft coils can reduce the pulsation on the surrounding tissue only if the neck of the aneurysm is completely sealed. Because most of these aneurysms typically have a broad neck, such complete sealing is very difficult if not impossible to achieve, resulting in the low observed efficacy of coil packing in relieving compression symptoms $s^{3,6,7}$ and the high rate of recanalization. ${ }^{3}$ Embolization by using liquid embolics may provide better long-term stability ${ }^{19}$; however, such embolization turns a soft pulsating mass into a hard volume that, even if completely occluding the aneurysm, transfers the pulsation of the parent artery to the surrounding tissue. ${ }^{20}$ Surgical treatment is considered superior in cases of compression syndromes because it is capable of immediate decompression of the neural tissue. However, surgery is made difficult by the large mass of the aneurysm, often requiring temporary clipping or proximal balloon occlusion and necessitating induced hypothermia or even cardiac arrest during surgery. ${ }^{21,22}$ Despite these difficulties, the efficacy of direct surgery in eliminating cranial nerve symptoms remains limited. ${ }^{23}$ Many of these aneurysms are repaired by bypass surgery rather than by clipping. ${ }^{24-28}$ While this technique can be performed without interrupting antegrade blood flow, the rate of morbiditymortality associated with the method is considerable. ${ }^{11}$ The limited success of conventional techniques justifies the search for safer and more effective treatment options. 
Flow diversion by using high-mesh-attenuation intraluminal endoprostheses, the newest advance in endovascular aneurysm therapy, has been recently introduced. ${ }^{13-16,29}$ The primary goal of diverting flow away from the aneurysm cavity is to allow the aneurysm to thrombose and the intima of the parent artery to cover the neck of the aneurysm once flow through the neck has ceased. Early reports suggest that in addition to better primary occlusion rates and improved long-term stability, this technique is capable of effectively eliminating mass effect and related compression symptoms. ${ }^{13}$ More recently, Piano et $\mathrm{al}^{30}$ reported shrinkage of the aneurysm following flow diversion in $61 \%$ of cases that were available for assessment.

In contrast to these favorable results, temporary aggravation of compression-related craniopathies may occur. ${ }^{14}$ In addition, delayed rupture of FD-treated large and giant aneurysms has been reported. ${ }^{31,32}$ In a voluntary self-audited survey conducted by the European Society of Minimally Invasive Neurologic Therapy, delayed rupture was found in $0.96 \%$ of 1421 aneurysms but in $2.1 \%$ of large and giant lesions. ${ }^{33}$ Flow diversion is further complicated by the necessity of aggressive antithrombotic treatment and potential thrombosis of the parent artery in the case of insufficient antiaggregation.

The present report focuses on the radiologic and clinical effects of endoluminal flow alteration on large and giant aneurysms treated solely by the FD technique. Cases were retrospectively selected from a consecutive series of 98 aneurysms that were treated with FD for various indications in our institution. Only aneurysms that were larger than $10 \mathrm{~mm}$ and treated without coils were included. The 6-month and 1-year angiographic occlusion rates were similar to those reported in other flow diversion studies, ${ }^{13-15}$ and clinical outcomes closely matched radiologic findings.

On posttreatment MR imaging follow-up, 27 of 30 aneurysms (90\%) collapsed completely. In another 3 cases (10\%), the aneurysm shrank but did not fully disappear. In 2 of those cases, image analysis disclosed a heavily calcified aneurysm wall (Fig 2C, $-D$ ). The rigid, calcified wall of the aneurysm may prevent or delay collapse even if there is no flow inside the sac. In 1 case, some shrinkage of the sac was first detected 8 months after treatment even though the aneurysm was completely occluded on angiography at 6 months. Despite every effort (including aggressive steroid treatment), the patient's visual acuity, which deteriorated 8 weeks after treatment, only started to improve at this time. The visual acuity finally improved from the pretreatment status of $0.4-0.25$ to $0.8-0.8$. In the other case, the role of wall calcification could not be evaluated because complete aneurysm occlusion was not achieved and the aneurysm had residual flow at 6-month angiography. In this case, no aneurysm shrinkage was detected and the sac did not lose its mixed high T1WI-T2WI signal intensity. This patient's visual acuity slightly deteriorated from 0.2 to 0.1 (Fig 2). In the third case, the ICA was occluded following bridging of a giant fusiform carotid cavernous aneurysm by using a long construct of PEDs. In this case, the size of the aneurysm decreased but it did not fully disappear, though the associated double vision had resolved.

In 27 cases, complete angiographic occlusion was achieved with the exception of a very small neck remnant. The aneurysms completely collapsed and became isointense. In all of these cases, the relevant cranial nerve compression symptoms either improved or resolved completely. All subjects in this series were treated in a single session, and none have been retreated.

FDs are thought to be effective by reducing blood flow through the aneurysm orifice. Similar to other stents, the implanted FD induces neointimal growth over its surface. Once blood flow entering and exiting the aneurysm has been substantially reduced, the neointimal layer may completely cover the orifice and seal the aneurysm from the parent artery. Reduced flow within the sac initiates thrombosis. During the acute stage of thrombosis, the aneurysm mass may increase in size, further increasing the associated mass effect ${ }^{34,35}$ and possibly leading to temporary aggravation of related clinical symptoms, as observed in 4 of 27 patients in our series, thereby necessitating the application of high-dose corticosteroids. The reduction of mass effect is expected by cessation of aneurysm pulsation and collapse of the mass of the aneurysm, which later requires thrombus organization that may take weeks to months following complete cessation of intra-aneurysmal flow. In our study, aneurysm shrinkage was seen as early as 3 months and as late as 8 months after treatment. Lack of aneurysm filling on angiography was paralleled by a reduction of aneurysm size on MR imaging in most (but not all) cases. Clinical symptoms, however, did not improve until the aneurysm started shrinking and losing its mixed signal intensity on MR imaging. Angiographic occlusion after FD treatment indicates redirection of flow into the parent artery but does not demonstrate completion of intra-aneurysmal thrombosis.

There were no delayed hemorrhagic complications reported in this series. Acute in-stent thrombosis occurred in 2 cases, both of which were successfully resolved by thrombolysis. The parent artery occluded during the subacute phase in 1 case and later recanalized completely. In-stent thrombosis was attributed to the patient's noncompliance in taking antiplatelets. An ICA occluded in a delayed fashion and could not be recanalized, which was attributed to premature cessation of clopidogrel 3 months after treatment of a giant and (more important) fusiform aneurysm. Such aneurysms likely require much longer times to endothelialize compared with berry aneurysms.

\section{CONCLUSIONS}

In this small series, FD treatment was highly effective in eliminating radiologic mass effect and resolving related clinical symptoms. Clinical improvement is expected only after aneurysm shrinkage or collapse. Angiographic occlusion indicates a lack of flow through the aneurysm orifice, but complete thrombosis may take considerably longer. MR imaging studies are strongly suggested in the follow-up of FD-treated aneurysms, especially when aneurysm-associated symptoms do not resolve.

\section{ACKNOWLEDGMENTS}

The authors gratefully thank Drs Aaron Berez and Peter K. Nelson for their invaluable help in the introduction of the technique in our institution and Janice $\mathrm{Ahn}, \mathrm{PhD}$, for her assistance in preparation of the manuscript. 
Disclosures: Istvan Szikora—RELATED: Grant: Covidien/ev3, ${ }^{*}$ National Science and Research Fund (OTKA), Hungary, ${ }^{*}$ Comments: Devices were provided by Covidien/ev3 for free as a scientific grant, No. T 73773, Consulting Fee or Honorarium: Covidien/ev3, Comments: proctoring and consulting agreement, Support for Travel to Meetings for the Study or Other Purposes: Covidien/ev3, UNRELATED: Board Membership: GE Healthcare, Comments: Medical Advisory Board, Consultancy: Stryker Neurovascular, Comments: proctoring and consulting agreement. *Money paid to the institution.

\section{REFERENCES}

1. Unruptured intracranial aneurysms: risk of rupture and risks of surgical intervention-International Study of Unruptured Intracranial Aneurysms Investigators. N Engl J Med 1998;339:1725-33

2. Wiebers DO, Whisnant JP, Huston J 3rd, et al. Unruptured intracranial aneurysms: natural history, clinical outcome, and risks of surgical and endovascular treatment. Lancet 2003;362:103-10

3. van Rooij WJ, Sluzewski M. Unruptured large and giant carotid artery aneurysms presenting with cranial nerve palsy: comparison of clinical recovery after selective aneurysm coiling and therapeutic carotid artery occlusion. AJNR Am J Neuroradiol 2008;29:997-1002

4. Lee T, Baytion M, Sciacca R, et al. Aggregate analysis of the literature for unruptured intracranial aneurysm treatment. AJNR Am J Neuroradiol 2005;26:1902-08

5. Raaymakers TW, Rinkel GJ, Limburg M, et al. Mortality and morbidity of surgery for unruptured intracranial aneurysms: a metaanalysis. Stroke 1998;29:1531-38

6. Halbach VV, Higashida RT, Dowd CF, et al. The efficacy of endosaccular aneurysm occlusion in alleviating neurological deficits produced by mass effect. J Neurosurg 1994;80:659-66

7. Sluzewski M, Menovsky T, van Rooij WJ, et al. Coiling of very large or giant cerebral aneurysms: long-term clinical and serial angiographic results. AJNR Am J Neuroradiol 2003;24:257-62

8. van der Schaaf IC, Brilstra EH, Buskens E, et al. Endovascular treatment of aneurysms in the cavernous sinus: a systematic review on balloon occlusion of the parent vessel and embolization with coils. Stroke 2002;33:313-18

9. de Gast AN, Sprengers ME, van Rooij WJ, et al. Midterm clinical and magnetic resonance imaging follow-up of large and giant carotid artery aneurysms after therapeutic carotid artery occlusion. Neurosurgery 2007;60:1025-29, discussion 1029-31

10. Brilstra EH, Rinkel GJ, Klijn CJ, et al. Excimer laser-assisted bypass in aneurysm treatment: short-term outcomes. J Neurosurg 2002;97:1029-35

11. Vajkoczy P, Korja M, Czabanka M, et al. Experience in using the excimer laser-assisted nonocclusive anastomosis nonocclusive bypass technique for high-flow revascularization: Mannheim-Helsinki series of 64 patients. Neurosurgery 2012;70:49-54, discussion 54-55

12. Lubicz B, Collignon L, Raphaeli G, et al. Flow-diverter stent for the endovascular treatment of intracranial aneurysms: a prospective study in 29 patients with 34 aneurysms. Stroke 2010;41:2247-53

13. Szikora I, Berentei Z, Kulcsar Z, et al. Treatment of intracranial aneurysms by functional reconstruction of the parent artery: the Budapest experience with the Pipeline embolization device. AJNR Am J Neuroradiol 2010;:1139-47

14. Lylyk P, Miranda C, Ceratto R, et al. Curative endovascular reconstruction of cerebral aneurysms with the Pipeline embolization device: the Buenos Aires experience. Neurosurgery 2009;64:632-42, discussion 642-43, quiz N6

15. Nelson PK, Lylyk P, Szikora I, et al. The Pipeline embolization device for the intracranial treatment of aneurysms trial. AJNR Am J Neuroradiol 2011;32:34-40

16. Fischer S, Vajda Z, Aguilar Perez M, et al. Pipeline embolization device (PED) for neurovascular reconstruction: initial experience in the treatment of 101 intracranial aneurysms and dissections. Neuroradiology 2012;54:369-82

17. Saatci I, Yavuz K, Ozer C, et al. Treatment of intracranial aneurysms using the Pipeline flow-diverter embolization device: a single-center experience with long-term follow-up results. AJNR Am J Neuroradiol 2012;33:1436-46

18. FDA Executive Summary P100018. Chestnut Medical. Pipeline Embolization Device. Prepared for the March 18, 2011 meeting of the
Neurologic Devices Panel. http://www.fda.gov/downloads/Advisory Committees/CommitteesMeetingMaterials/MedicalDevices/Medical DevicesAdvisoryCommittee/NeurologicalDevicesPanel/UCM247161. pdf, 2011. Accessed August 31, 2011

19. Molyneux AJ, Cekirge S, Saatci I, et al. Cerebral Aneurysm Multicenter European Onyx (CAMEO) trial: results of a prospective observational study in 20 European centers. AJNR Am J Neuroradiol 2004;25:39-51

20. Van Loock K, Menovsky T, Voormolen MH, et al. Microsurgical removal of Onyx HD-500 from an aneurysm for relief of brainstem compression: case report. J Neurosurg 2010;113:770-73

21. Taki W, Sakai N, Nakahara I, et al. Circulatory arrest with profound hypothermia during the surgical treatment of large internal carotid artery aneurysm: case report. Neurol Med Chir (Tokyo) 1998;38: 25-29

22. Barzó P, Bogáts G, Babik B, et al. Surgical treatment of giant basilar artery aneurysm with induced hypothermia and circulatory arrest [in Hungarian]. Orv Hetil 2001;142:2747-52

23. Dehdashti AR, Le Roux A, Bacigaluppi S, et al. Long-term visual outcome and aneurysm obliteration rate for very large and giant ophthalmic segment aneurysms: assessment of surgical treatment. Acta Neurochir (Wien) 2012;154:43-52

24. Iihara K, Okawa M, Hishikawa T, et al. Slowly progressive neuronal death associated with postischemic hyperperfusion in cortical laminar necrosis after high-flow bypass for a carotid intracavernous aneurysm. J Neurosurg 2010;112:1254-59

25. Kan P, Liu JK, Couldwell WT. Giant fusiform aneurysm in an adolescent with PHACES syndrome treated with a high-flow external carotid artery-M3 bypass: case report and review of the literature. J Neurosurg 2007;106(6 suppl):495-500

26. Reinert M, Barth A, Schroth G, et al. Repeated laser-assisted highflow bypass for recurrent giant intracranial aneurysm. Swiss Med Wkly 2006;136:353-56

27. Streefkerk HJ, Wolfs JF, Sorteberg W, et al. The ELANA technique: constructing a high flow bypass using a non-occlusive anastomosis on the ICA and a conventional anastomosis on the SCA in the treatment of a fusiform giant basilar trunk aneurysm. Acta Neurochir (Wien) 2004;146:1009-19, discussion 1019

28. Liebig T, Henkes H, Fischer S, et al. Fibered electrolytically detachable platinum coils used for the endovascular treatment of intracranial aneurysms: initial experiences and mid-term results in 474 aneurysms. Interv Neuroradiol 2004;10:5-26

29. Byrne JV, Beltechi R, Yarnold JA, et al. Early experience in the treatment of intra-cranial aneurysms by endovascular flow diversion: a multicentre prospective study. PLoS One 2010;5:pii:e12492

30. Piano M, Valvassori L, Quilici L, et al. Midterm and long-term follow-up of cerebral aneurysms treated with flow diverter devices: a single-center experience. J Neurosurg 2013;118:408-16

31. Kulcsár Z, Houdart E, Bonafé A, et al. Intra-aneurysmal thrombosis as a possible cause of delayed aneurysm rupture after flow-diversion treatment. AJNR Am J Neuroradiol 2011;32:20-5

32. Turowski B, Macht S, Kulcsár Z, et al. Early fatal hemorrhage after endovascular cerebral aneurysm treatment with a flow diverter (SILK-stent): do we need to rethink our concepts? Neuroradiology 2011;53:37-41

33. Kulcsár Z, Szikora I, The ESMINT Retrospective Analysis of Delayed Aneurysm Ruptures after flow diversion (RADAR) study. http:// www.ejmint.org/original-article/1244000088. Accessed February 22, 2013

34. Hammoud D, Gailloud P, Olivi A, et al. Acute vasogenic edema induced by thrombosis of a giant intracranial aneurysm: a cause of pseudostroke after therapeutic occlusion of the parent vessel. $A J N R$ Am J Neuroradiol 2003;24:1237-9

35. Strother CM, Eldevik P, Kikuchi Y, et al. Thrombus formation and structure and the evolution of mass effect in intracranial aneurysms treated by balloon embolization: emphasis on MR findings. AJNR Am J Neuroradiol 1989;10:787-96 